\title{
In vitro Evaluation of the Location of Cholesteryl Ester Deposits on Monthly Replacement Silicone Hydrogel Contact Lens Materials
}

This article was published in the following Dove Press journal: Clinical Ophthalmology

\author{
Han Qiao' \\ Doerte Luensmann' \\ Miriam Heynen (1D' \\ Elizabeth Drolle' \\ Lakshman N Subbaraman' \\ Charles Scales ${ }^{2}$ \\ Donald Riederer ${ }^{2}$ \\ Zohra Fadli ${ }^{2}$ \\ Lyndon Jones $\mathbb{D}^{1,3}$ \\ 'Centre for Ocular Research \& Education \\ (CORE), School of Optometry \& Vision \\ Science, University of Waterloo, \\ Waterloo, ON, Canada; ${ }^{2}$ Johnson \& \\ Johnson Vision Care, Inc, Jacksonville, FL, \\ USA; ${ }^{3}$ Centre for Eye \& Vision Research \\ (CEVR), Hong Kong, People's Republic of \\ China
}

Correspondence: Doerte Luensmann Email dluensma@uwaterloo.ca
Purpose: The deposition profile of cholesteryl ester on the surface and throughout the matrix of silicone hydrogel contact lens (CL) materials was determined under conditions that mimic a daily wear regimen.

Methods: In this in vitro study, four SiHy CL materials (senofilcon C, lotrafilcon B, comfilcon A and samfilcon A) were incubated in an artificial tear solution (ATS) for up to 30 days. CL incubation was alternated between the ATS (16 hours) and a multipurpose care regimen ( 8 hours). The ATS included fluorescently tagged cholesteryl ester (5-cholesten-3ßol 6-[(7-nitro-2-1,3-benzoxadiazol-4-yl)amino]caproate; CE-NBD) and confocal laser scanning microscopy visualized the distribution of the lipid through the CLs.

Results: The distribution of CE-NBD was homogenous from the anterior to posterior surface in senofilcon $\mathrm{C}$ and comfilcon $\mathrm{A}$, at all time points. For lotrafilcon $\mathrm{B}$ and samfilcon A, CE-NBD localization was heterogeneous, with greater amounts on the surfaces on Day 1 and Day 14 compared to the lens matrix; however, differences in concentration between the surface and bulk diminished by Day 30 .

Conclusion: The distribution of the non-polar lipid CE-NBD varied with lens material chemistry. While some lens materials deposited the lipid primarily on the surface after 16 hours of exposure, all materials exhibited a homogenous distribution after one month.

Keywords: lipid distribution, silicone hydrogel contact lenses, cholesteryl ester, artificial tear solution

\section{Introduction}

Discomfort experienced during contact lens (CL) wear continues to be an issue, particularly towards the end of the day, ${ }^{1,2}$ and has been linked closely to $\mathrm{CL}$ discontinuation. ${ }^{3,4}$ The causes attributed to CL discomfort are multifactorial, with lens deposition being one of the potential factors. ${ }^{5}$ The degree to which tear film components deposit on CLs is primarily driven by the lens material and frequency of replacement, but other factors such as ocular physiology, tear film composition, wear modality, cleaning regimen, and environmental factors can all play a role in controlling the amount of deposition. ${ }^{6-8}$ Although protein deposition has been extensively studied, ${ }^{8}$ more attention over the past decade has been given to studying lipid deposition, particularly on silicone hydrogel materials. ${ }^{7,9-11}$ The composition and amount of lipid deposition have been characterized from both in vivo and in vitro studies. ${ }^{7,10-15}$ However, the location of this lipid deposition in terms of whether it is surface located or occurs within the bulk of the material is not fully understood. 9,16 
The location of protein deposition has been previously studied using confocal microscopy and sectioning methods. ${ }^{17}$ Accurately locating the lipid deposition profile throughout the lens may help to better understand its role in CL discomfort, because lipids deposited on the lens surfaces interact directly with the ocular surface and may be more prone to degradation through oxidative processes, which could contribute to downstream pathways leading to discomfort. ${ }^{18-20}$ Previous clinical studies have demonstrated little to no correlation between the quantity of lipid deposition and levels of discomfort. ${ }^{18-20}$ It is therefore beneficial to study the location of lipid deposition in addition to the quantity and composition of lipid deposition. The nonpolar phase of the lipid layer of the tear film is composed of cholesteryl esters, wax esters, free cholesterol, hydrocarbons and triglycerides. ${ }^{21}$ A representative lipid that makes up an abundant amount of the lipid layer that is believed to be vital in maintaining the structural integrity of the tear film is cholesterol oleate, which is part of the cholesteryl ester family. ${ }^{22}$ Large quantities of hydrophobic chains of cholesteryl esters allow for self-assembly of liquid-crystal-like structures that contribute to the stability of the tear film and will therefore be the focus of this study. $^{21}$

Reusable daily wear silicone hydrogel lenses, which include biweekly and monthly replacement lenses, account for approximately $52 \%$ of fits today. ${ }^{23}$ In the current study, the lipid deposition profile throughout the lens matrix was determined over a period of 30 days for four contemporary frequent replacement silicone hydrogel lens materials
(Table 1). An in vitro approach was used that further included a daily cleaning cycle, using a commonly prescribed multipurpose solution.

\section{Materials and Methods}

Four monthly replacement silicone hydrogel lenses were examined: Lotrafilcon B [Air Optix ${ }^{\circledR}$ Aqua, Alcon, Fort Worth, TX], comfilcon A [Biofinity ${ }^{\circledR}$, CooperVision, Pleasanton, CA], samfilcon A [Ultra ${ }^{\circledR}$, Bausch + Lomb, Rochester, NY], and senofilcon C $\left[\right.$ Acuvue $^{\circledR}$ Vita $^{\mathrm{TM}}$, Johnson \& Johnson Vision, Jacksonville, FL]. All lenses were obtained from the manufacturer in the original packaging and had a dioptric power of $-3.00 \mathrm{D}$. The lens properties are outlined in Table 1.

An artificial tear solution (ATS) was prepared using a slightly modified composition as described previously to incorporate the fluorescently tagged cholesteryl ester. ${ }^{24}$ The ATS included various physiological tear film components, such as different proteins, lipids and salts (Table 2). In comparison to the previously used ATS a slightly higher concentration of polar lipids was used by adding phosphatidylethanolamine. This was done to mimic the ratio of approximately $30: 70^{25}$ for polar vs non-polar lipids in the tear film a concentration of $0.0171 \mathrm{mg} / \mathrm{mL}$ for the combined polar lipids and $0.0394 \mathrm{mg} / \mathrm{mL}$ for the combined non-polar lipids was used. The ratio of Phosphatidylcholine to Phosphatidylethanolamine was 2:1 (comparable to Rantamäki, ${ }^{26}$ ratio 3.5:1) and the ratio of Cholesteryl oleate to Cholesterol was 13:1 (comparable to McMahon, ${ }^{27}$ ratio $10: 1$ ). To detect the lipid of interest, $0.1 \%$ of fluorescently

Table I Properties of Silicone Hydrogel Contact Lenses

\begin{tabular}{|c|c|c|c|c|}
\hline & Acuvue $^{\circledR}$ Vita $^{\text {TM }}$ & Air Optix ${ }^{\circledR}$ Aqua & Biofinity ${ }^{\circledR}$ & Ultra $^{\circledR}$ \\
\hline $\begin{array}{l}\text { United States Adopted Name } \\
\text { (USAN) }\end{array}$ & Senofilcon C & Lotrafilcon B & Comfilcon A & Samfilcon A \\
\hline Manufacturer & $\begin{array}{l}\text { Johnson \& } \\
\text { Johnson }\end{array}$ & Alcon & CooperVision & $\begin{array}{l}\text { Bausch + } \\
\text { Lomb }\end{array}$ \\
\hline Centre Thickness (mm) & 0.07 & 0.08 & 0.08 & 0.07 \\
\hline Water Content (\%) & 41 & 33 & 48 & 46 \\
\hline Oxygen Permeability $\left(x \mid 0^{-11}\right)$ & 122 & 110 & 128 & 114 \\
\hline Oxygen Transmissibility $\left(\times 10^{-9}\right)$ & 147 & 138 & 160 & 163 \\
\hline Surface Treatment & None & Plasma Coating & None & None \\
\hline Wetting Agent & PVP & $\begin{array}{l}\text { Wetting agent in packaging solution ( } 1 \% \text { copolymer } \\
845 \text { ) }\end{array}$ & None stated & PVP \\
\hline
\end{tabular}


Table 2 Composition of Artificial Tear Solution

\begin{tabular}{|c|c|c|c|c|c|c|c|}
\hline $\begin{array}{l}\text { Organic/Inorganic } \\
\text { Components }\end{array}$ & $\begin{array}{l}\mathrm{mg} / \\
\mathrm{mL}\end{array}$ & Supplier & $\begin{array}{l}\text { Catalogue } \\
\text { Number }\end{array}$ & Lipid Components & $\begin{array}{l}\mathrm{mg} / \\
\mathrm{mL}\end{array}$ & Supplier & $\begin{array}{l}\text { Catalogue } \\
\text { Number }\end{array}$ \\
\hline Sodium chloride & 5.26 & EMD & SX0420-3 & Cholesteryl Oleate & 0.03669 & Sigma-Aldrich & C9253 \\
\hline Potassium chloride & 1.19 & $\begin{array}{l}\text { Sigma- } \\
\text { Aldrich }\end{array}$ & P5405 & Cholesteryl ester NBD & 0.00003 & $\begin{array}{l}\text { Avanti Polar } \\
\text { Lipids }\end{array}$ & $81025 \mathrm{IP}$ \\
\hline Sodium carbonate & 1.27 & $\begin{array}{l}\text { Sigma- } \\
\text { Aldrich }\end{array}$ & 223484 & Cholesterol & 0.00275 & Sigma-Aldrich & C8667 \\
\hline Potassium hydrogen carbonate & 0.30 & $\begin{array}{l}\text { Sigma- } \\
\text { Aldrich }\end{array}$ & 237205 & Phosphatidylethanolamine & 0.00592 & Sigma-Aldrich & 76548 \\
\hline Trisodium citrate dihydrate & 0.44 & $\begin{array}{l}\text { Sigma- } \\
\text { Aldrich }\end{array}$ & SI804 & Phosphatidylcholine & 0.01116 & Sigma-Aldrich & P3556 \\
\hline Calcium chloride dihydrate & 0.07 & $\begin{array}{l}\text { Sigma- } \\
\text { Aldrich }\end{array}$ & C7902 & Protein components & $\begin{array}{l}\mathrm{mg} / \\
\mathrm{mL}\end{array}$ & Supplier & Catalogue number \\
\hline Urea & 0.07 & $\begin{array}{l}\text { Sigma- } \\
\text { Aldrich }\end{array}$ & U5378 & Bovine albumin & 0.20 & Sigma-Aldrich & A7888 \\
\hline Glucose & 0.04 & $\begin{array}{l}\text { Sigma- } \\
\text { Aldrich }\end{array}$ & G702I & Hen Egg Lysozyme & 1.90 & Sigma-Aldrich & L6876 \\
\hline Sodium phosphate dibasic & 3.41 & $\begin{array}{l}\text { Sigma- } \\
\text { Aldrich }\end{array}$ & S7907 & Bovine mucin & 0.15 & Sigma-Aldrich & M3895 \\
\hline Hydrochloric acid & $\begin{array}{l}\mathrm{pH} \text { to } \\
7.4\end{array}$ & $\begin{array}{l}\text { Sigma- } \\
\text { Aldrich }\end{array}$ & H9892 & $\begin{array}{l}\text { Recombinant Human } \\
\text { Lactoferrin }\end{array}$ & 1.80 & InVitria & 777LAC015 \\
\hline Bacteriostatic Agent & $\mathbf{V} / \mathbf{V} \%$ & Supplier & Catalogue Number & & & & \\
\hline ProClin 300 & 0.02 & $\begin{array}{l}\text { Sigma- } \\
\text { Aldrich }\end{array}$ & $48912-U$ & & & & \\
\hline
\end{tabular}

Abbreviation: PVP, polyvinyl pyrrolidone.

tagged cholesteryl ester (5-cholesten-3ß-ol 6-[(7-nitro2-1,3-benzoxadiazol-4-yl)amino]caproate - CE-NBD) was added. The biocide ProClin 300 was included in the ATS to prevent bacterial contamination. The osmolality and surface tension of the ATS used in this study were within the same ranges as previously reported (osmolality: $304-306 \mathrm{mmol} /$ $\mathrm{kg}$, surface tension: 40-46 dynes $/ \mathrm{cm}){ }^{24}$

Daily lens wear was simulated for 1 day, 14 days and 30 days. Prior to the incubation in ATS, lenses were removed from the original manufacturer's packaging and soaked in $2 \mathrm{~mL}$ of phosphate buffer saline (PBS) (EMDMillipore, Billerica, MA) for 17 hours using a 24 well polystyrene plate (Corning ${ }^{\circledR}$, Corning, NY) to remove residual blister pack solution. This procedure was followed since the composition of the blister solution is unknown, but will differ from company to company, ${ }^{28}$ and thus could potentially make the interpretation of the results more complex and potentially confounding. Lenses were then blotted on lens paper and transferred into a $6 \mathrm{~mL}$ glass vial
(Wheaton, Millville, NJ) containing $1 \mathrm{~mL}$ of ATS for 16 hrs in a $37^{\circ} \mathrm{C}$ incubator, while rotated at $60 \mathrm{rpm}$. One milliliter of ATS was required to submerge the lens in the vial study. A moderate rotation setting of $60 \mathrm{rpm}$ was selected to simulate the non-stagnant wear experienced when a patient is wearing lenses. Day 1 lenses were removed from the ATS and prepared for imaging as described below, while day 14 and day 30 lenses were transferred to a 24 well plate containing $2 \mathrm{~mL}$ of OPTIFREE $^{\circledR}$ PureMoist $^{\circledR}$ care solution (OPTI-FREE PM, Alcon, Fort Worth, TX) to simulate a daily cleaning regime without applying any manual lens rubbing to reduce variability. After 8 hours at room temperature, lenses were placed back into freshly prepared ATS. This lens cycling was repeated between the care solution and ATS on each weekday for 14 and 30 days, respectively. Over the weekends, lenses remained incubated in ATS without cycling. Control CLs were incubated in ATS without CE-NBD. To reduce transfer of excess ATS and care 


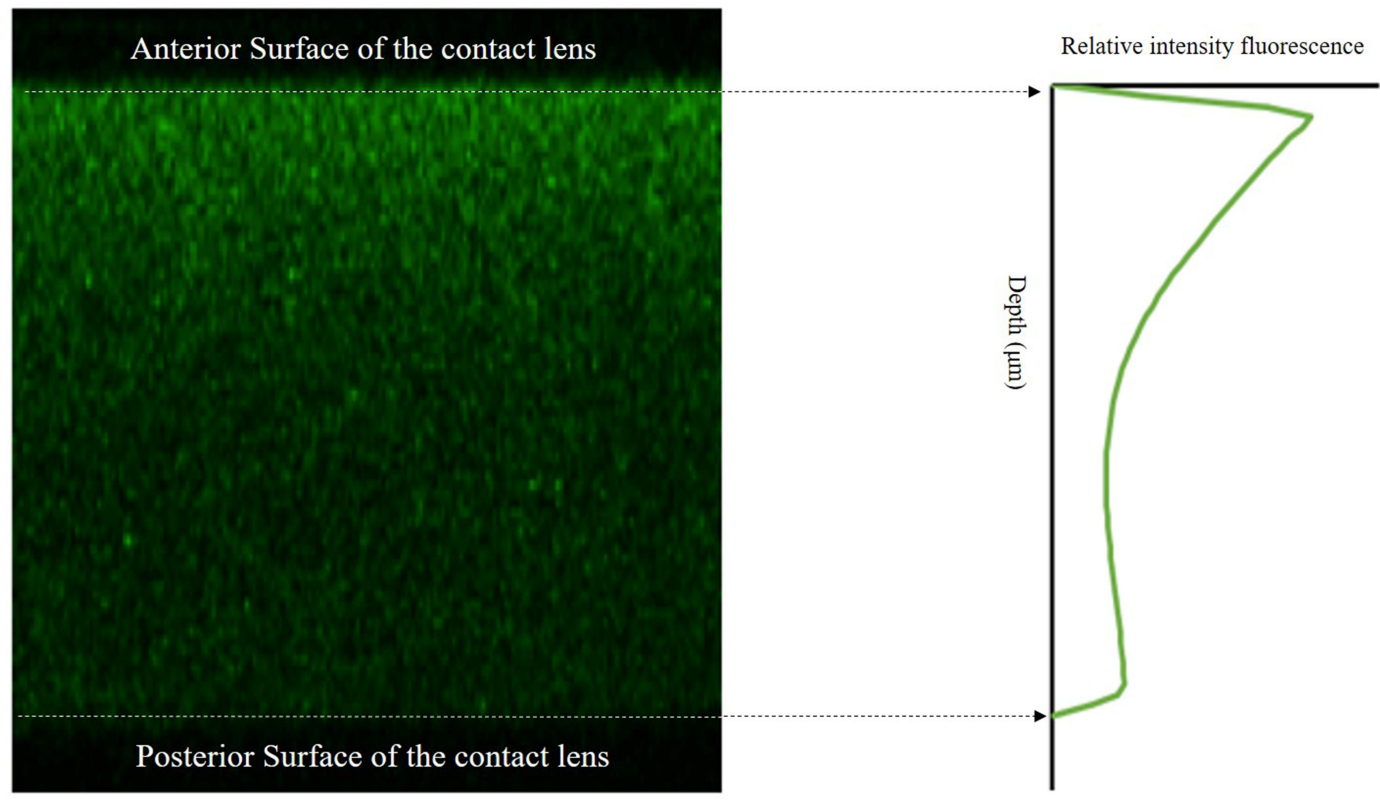

Figure I Schematic depiction of a cross-section through a contact lens. The relative intensity fluorescence (RIF) describes the location of CE-NBD from the anterior to the posterior surface of the lens. Image not to scale.

solution, lenses were rinsed in $2 \mathrm{~mL}$ of PBS three times after each incubation. At the respective time points, lenses were removed from ATS and prepared for CLSM imaging. A $5 \mathrm{~mm}$ disk was punched out from the centre of the lens and fixed onto a microscope slide (Goldline, VWR, Radnor, PA) as described previously. ${ }^{17}$ Four replicates were analyzed for each lens type at each time point.

The lens samples were imaged using a Zeiss LSM 510 Meta, Axiovert 200 confocal microscope (ZEISS Inc., Toronto, Canada) from the anterior to posterior surface of the lens at three different locations (Figure 1). To visualize the CE-NBD deposited in the lens, the excitation was set to $488 \mathrm{~nm}$ on an Argon laser and the emission wavelength was set to $505 \mathrm{~nm}$ using a long-pass filter. The lens samples were viewed under a C-Apochromat 40X/1.2 corr objective lens, which magnified the lens such that each visible plane was $224.56 \mu \mathrm{m}^{2.17}$ The laser setting (gain setting on the photomultiplier detector) was adjusted if necessary to capture the dynamic range of the fluorescence intensity over four lens types at three incubation time points. The deposition profile at each plane along the depth of the lens was scanned at $2 \mu \mathrm{m}$ intervals using z-stacking and were rendered into a crosssectional image of the lens using ZEN Lite software (ZEISS Inc., Germany).

For the image analysis, the average relative intensity of fluorescence (RIF) value at each plane of the lens was plotted against the depth of the lens using ImageJ software (Bethesda,
MS, USA) to graph the corresponding deposition profile curve (Figure 1). Since each lens profile had a slightly different thickness, all scans were normalized to the equal number of data points using piecewise linear interpolation. The RIF values imaged at the three locations were averaged and the values were then averaged with the four replicates of each lens type at each time point. The endogenous fluorescence intensity of the negative control CLs was subtracted from the test CLs. In addition, to compare the data between the different incubation periods, a scaling factor was calculated to account for the different gain settings used to capture the images on day 30 . The scaling factor was determined by imaging the 14-day CLs at both the 14-day gain setting as well as the 30-day gain setting. A linear relationship between gain and fluorescence intensity for the range of gain settings was confirmed.

Statements on comparisons between the different lens deposition profiles and time points are intended to allow for easier interpretation of the data and are not based on inferential statistical analysis. Since the aim of the study was to visualize CE-NBD throughout the lens and was not intended to quantify the amount deposited, additional statistical analysis was not conducted.

\section{Results}

All materials accumulated CE-NBD over time and the deposition profile varied depending on the duration of incubation and the lens type (Figure 2A-D). 


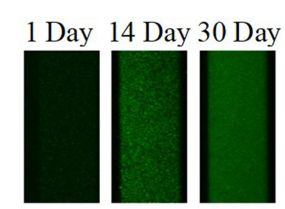

A senofilcon C

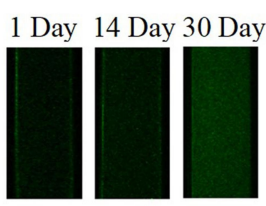

B lotrafilcon B

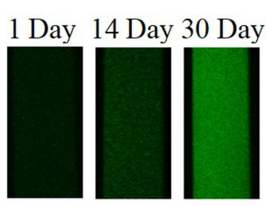

C comfilcon A

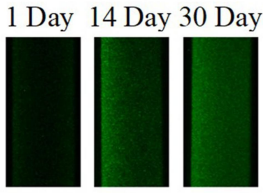

D samfilcon A

Figure 2 Example images of CE-NBD fluorescence profiles through senofilcon C (A), lotrafilcon B (B), comfilcon A (C), and samfilcon A (D) over 30 days.

A

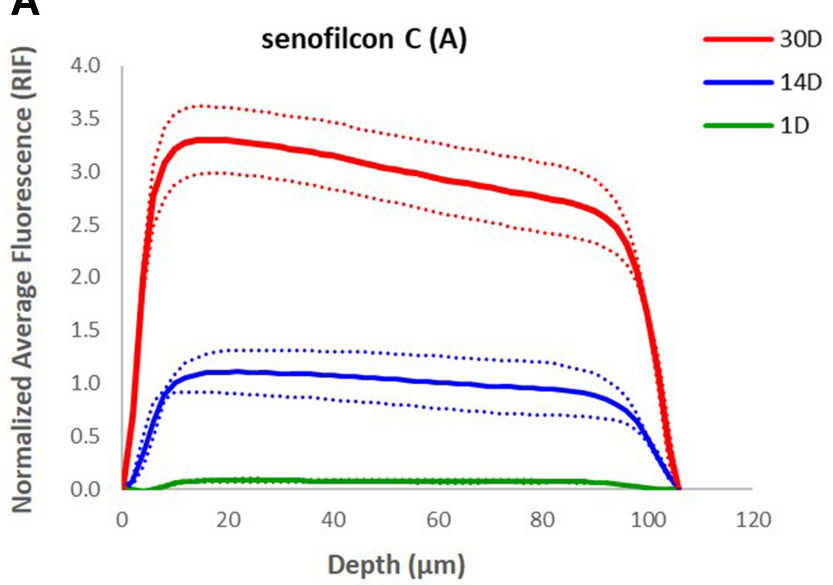

C

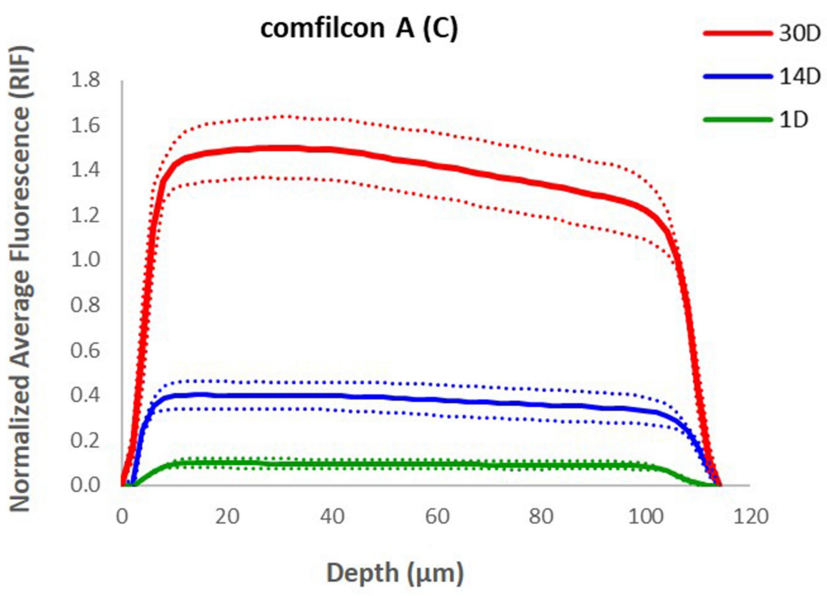

B

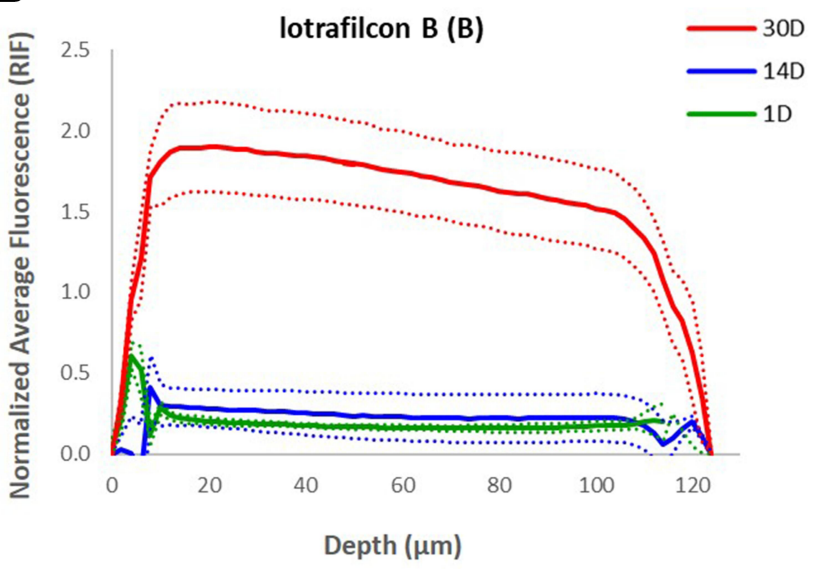

D

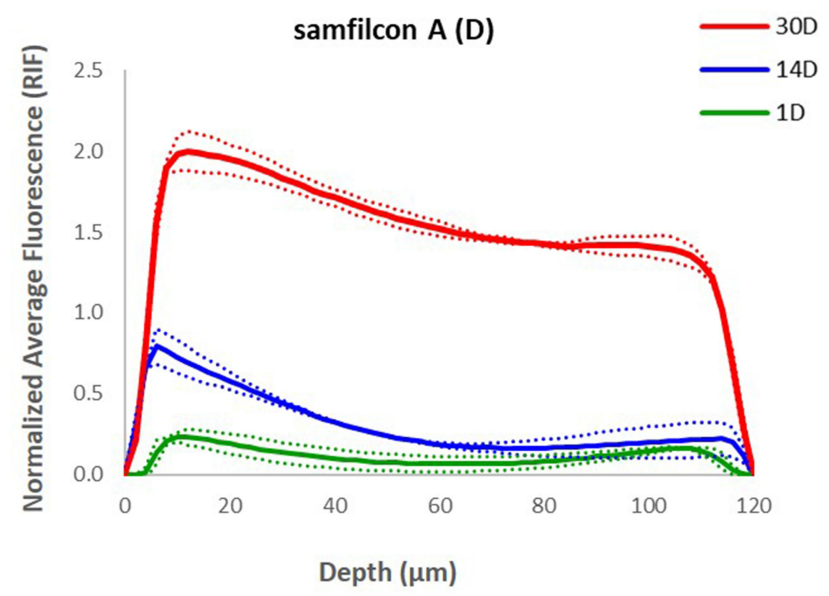

Figure 3 CE-NBD deposition profiles of senofilcon C (A), lotrafilcon B (B), comfilcon A (C), samfilcon A (D) lenses after I, I4, and 30 days of incubation. The 30-day scaled curves were obtained by multiplying the 30-day RIF (relative intensity fluorescence) with a scaling factor of 3.3, I.9, I.5, and 2 for senofilcon C, lotrafilcon B, comfilcon $A$, and samfilcon A, respectively. The standard deviation values are shown as dotted lines in the corresponding colour. The $x$-axis shows the normalized depth ( $\mu \mathrm{m}$ ) from the anterior to the posterior surface of the lens, the Y-axis (RIF) shows the intensity of the emitted fluorochrome.

After 1 day of incubation, the deposition profile was uniform in senofilcon $\mathrm{C}$ and comfilcon A materials, whereas lotrafilcon B and samfilcon A had higher RIF values on the surfaces than the core of the lens (Figure 3A-D). In samfilcon A, the RIF value became gradually lower towards the centre of the lens, while lotrafilcon B exhibited distinct fluorescent peaks on both surfaces with uniformly lower RIF values distributed throughout the matrix.
After 14 days of incubation, lenses exhibited a higher RIF signal in the lens matrix, indicating more lipid uptake compared to Day 1 (Figure 3A-D). The uptake pattern for senofilcon $\mathrm{C}$ and comfilcon was similar compared to day 1. For samfilcon A, higher RIF values were observed at the anterior and posterior surfaces of the lens compared to the center of the lens. Moreover, the RIF value at the posterior surface of samfilcon A was lower than the anterior surface. 
The gradual decrease of the RIF signal when traveling through the lens material and resulting asymmetry between surfaces is due to laser attenuating as shown previously $^{29}$ and not an indicator of lower deposition rates on the back surface compared to front surface. For lotrafilcon $\mathrm{B}$, the peaks at both surfaces were slightly less pronounced compared to Day 1.

After 30 days of incubation, the adjusted RIF signal was significantly higher for all lenses on Day 30 compared to Day 14 after the scaling factor was applied, confirming a continuous increase in CE-NBD accumulation throughout the 30 days. The uniform deposition curves suggest that CE-NBD was evenly distributed in all lens types, with the exception of samfilcon A (Figure 3A-D). Although the curve suggests a more homogeneous distribution pattern than at the previous incubation periods, samfilcon A maintained a slightly higher RIF value at the anterior and posterior surfaces compared to the center of the lens after 30 days of incubation.

All CE-NBD deposition profile curves exhibited an apparent asymmetry between the anterior and posterior surfaces, with higher RIF values on the anterior surfaces than the posterior surfaces. This phenomenon was also observed in previous studies conducted using the same confocal technique. ${ }^{17}$ To determine whether this was a true effect, additional experiments were conducted, which imaged the lenses in the reverse orientation from the posterior to anterior surface, at the three time points (data not shown). The same reduction in intensity was noted the deeper the laser traveled through the lens material. This suggests that the asymmetry in the RIF values between the two surfaces is due to depth-dependent attenuation of the laser through the thickness of the CL. ${ }^{29}$

\section{Discussion}

Upon the insertion of a CL, various tear components readily deposit onto the lens. ${ }^{7,30}$ These deposits can alter the lens property and its interaction with the ocular surface and tear film, ${ }^{31,32}$ which may impact the lens performance on eye. ${ }^{19}$ Previous studies have visualized surface and bulk lipid deposition using various lipid stains, such as Nile Red and oil red O. ${ }^{16,33}$ However, contrasting results were observed in the stain behavior between different studies ${ }^{16,33}$ and it has been suggested that lipid stains may produce inconsistent staining that is not reflective of actual lipid deposits within the lens. ${ }^{34}$ In the current study, CE-NBD was used as a spectroscopically traceable analogue to determine $\mathrm{CO}$ uptake. CE-NBD and CO have a similar molecular weight
(MW 662.9 vs 651.1) and the NBD moiety and oleic acid are both esterified to cholesterol at C3. ${ }^{35,36} \mathrm{CE}-\mathrm{NBD}$-cholesterol is only slightly less lipophilic than $\mathrm{CO}$, but more lipophilic than cholesterol, as defined by computed partition coefficients $\left(10.9,16.6,8.7\right.$, respectively). ${ }^{35,36}$

Regardless of lens type, the amount of CE-NBD deposition increased with time, as suggested by an increased RIF value (Figure 3A-D). Although a cleaning regimen was used, lipid deposition continued to increase with time. The location of the cholesteryl ester deposits was highly influenced by the lens material. The lenses containing NVP (comfilcon A) and PVP (senofilcon C) showed a strong attraction of cholesteryl ester into the lens matrix, which may suggest that NVP within a lens can enhance lipid incorporation, by increasing lipid solubility within the matrix. ${ }^{37}$ Samfilcon A also incorporates PVP monomers; however, a more gradual increase in lipid accumulation towards the lens centre was seen in this lens type, suggesting that either the PVP distributed within the lens material is different from that seen in senofilcon A or the concentration relative to the other material components differs between the two materials. Lotrafilcon B, which has a plasma surface treatment, deterred cholesteryl ester deposition to the bulk of the lens for the first 14 days and mainly deposited on the surfaces. ${ }^{38}$ This observation may be compared to the slightly lower amount of lipid deposited in lotrafilcon B than other CLs seen in previous studies. ${ }^{12,14}$ This suggests that surface properties may play a bigger role in reducing overall lipid deposition than bulk polymeric composition.

However, regardless of the type of silicone hydrogel tested, the amount of lipids deposited increased over time, which is in agreement with studies investigating the quantitative uptake to silicone hydrogel lenses. ${ }^{715}$ Whether or not higher lipid uptake over time has any impact on CL comfort is still unclear and requires further clinical investigation. ${ }^{18,20}$

CE-NBD was imaged using confocal microscopy, which is a useful tool to detect tear film component deposition at different depths within the lens material. However, a few limitations should be mentioned. In this study, the range of fluorescence intensity over the course of 30 days was too large and required the adjustment of gain settings, and scaling factors had to be used to encompass both the low RIF at Day 1 and high RIF at Day 30. In addition, it is unclear if the quantum yield of the fluorophore may differ depending on the lens material or if the sorption properties of NBD-CE may be slightly different compared to unconjugated $\mathrm{CE} .{ }^{39}$ For this reason, the RIF 
values cannot be used to determine the relative amount of deposition between different lens materials.

This was the first study to determine the location of non-polar lipids throughout the matrix of contact lenses using a complex ATS and a daily cleaning cycle to better mimic typical wear conditions over one month. However, since a static vial with a set volume of solution was used during the incubation phases, lenses were permanently immersed in ATS. This does not accurately reflect the interactions between the lens and the ocular environment, including factors such as tear flow, tear exchange, intermittent air exposure, and friction due to blinking. Whether this has an impact on the lipid uptake was recently investigated by Walther et al, ${ }^{40}$ who compared the lipid accumulation on daily disposable lenses. The authors concluded that the vial method may result in a faster uptake of the fluorescently labeled lipid for certain lens materials compared to an in vitro eye-blink instrument, which simulates physiologic tear flow ( $2 \mathrm{~mL} / 24$ hours). ${ }^{40}$ Moreover, the cleaning regime did not incorporate a rubbing step to minimize variability. Even though previous studies on tear film deposits have found no effect or only a minor difference in uptake between lenses that were cleaned with and without lens rubbing, ${ }^{41,42}$ there is the potential that this may have impacted the distribution of lipids on the lenses over time. Future in vitro studies on contact lens deposition may therefore benefit if additional features of the ocular environment/anatomy and lens care regime can be simulated.

\section{Conclusion}

As seen with tear film proteins, ${ }^{17}$ the lipid cholesteryl ester deposited on the surface and within the lens matrix of all four silicone hydrogel lens materials over time and varied depending on the lens material. These different lipid deposition profiles may have varying effects, such that lens surface deposits interact more readily with the ocular surface, versus a limited interaction of lipids sequestered within the bulk of the lens and further studies on the clinical relevance of this is warranted.

\section{Funding}

Funding for this study was provided by Johnson \& Johnson Vision.

\section{Disclosure}

Over the past 3 years, H. Qiao, D. Luensmann, M. Heynen, E. Drolle, L.N. Subarraman and L. Jones at CORE have received research funding and/or honoraria from the following companies: Alcon, Allergan, Contamac, CooperVision, GL Chemtech, Johnson \& Johnson Vision, SightGlass, Lubris, Menicon, Nature's Way, Novartis, Ote, Ophtecs, PS Therapy, Santen, Shire, TearScience and Visioneering. L. Subbaraman was employed at CORE while the study was conducted and is currently employed at Alcon Laboratories, Inc. C. Scales, D. Riederer and Z. Fadli are currently employed at Johnson \& Johnson Vision. The authors report no other conflicts of interest in this work.

\section{References}

1. Nichols KK, Redfern RL, Jacob JT, et al. The TFOS International Workshop on Contact Lens Discomfort: report of the definition and classification subcommittee. Invest Ophthalmol Vis Sci. 2013;54: TFOS14-9. doi:10.1167/iovs.13-13074

2. Chalmers R. Overview of factors that affect comfort with modern soft contact lenses. Cont Lens Anterior Eye. 2014;37:65-76. doi:10.1016/ j.clae.2013.08.154

3. Richdale K, Sinnott LT, Skadahl E, et al. Frequency of and factors associated with contact lens dissatisfaction and discontinuation. Cornea. 2007;26:168-174. doi:10.1097/01.ico.0000248382.32143.86

4. Dumbleton K, Woods CA, Jones LW, et al. The impact of contemporary contact lenses on contact lens discontinuation. Eye Contact Lens. 2013;39:93-99. doi:10.1097/ICL.0b013e318271caf4

5. Nichols JJ, Willcox MD, Bron AJ, et al. The TFOS International Workshop on Contact Lens Discomfort: executive summary. Invest Ophthalmol Vis Sci. 2013;54:TFOS7-TFOS13. doi:10.1167/iovs.13-13212

6. Jones L, Brennan NA, Gonzalez-Meijome J, et al. The TFOS International Workshop on Contact Lens Discomfort: report of the contact lens materials, design, and care subcommittee. Invest Ophthalmol Vis Sci. 2013;54:TFOS37-70. doi:10.1167/iovs.1313215

7. Walther H, Lorentz H, Heynen M, et al. Factors that influence in vitro cholesterol deposition on contact lenses. Optom Vis Sci. 2013;90:1057-1065. doi:10.1097/OPX.0000000000000022

8. Luensmann D, Jones L. Protein deposition on contact lenses: the past, the present, and the future. Cont Lens Anterior Eye. 2012;35:53-64. doi:10.1016/j.clae.2011.12.005

9. Jacob J, Frederick R, Tucker C, et al. Effect of static and non-static in-vitro techniques on lipid penetration into SIHy contact lenses. Invest Ophth Vis Sci. 2013;54:5486.

10. Heynen M, Lorentz H, Srinivasan S, et al. Quantification of non-polar lipid deposits on senofilcon a contact lenses. Optom Vis Sci. 2011;88:1172-1179. doi:10.1097/OPX.0b013e31822a5295

11. Lorentz H, Heynen M, Trieu D, et al. The impact of tear film components on in vitro lipid uptake. Optom Vis Sci. 2012;89:856-867.

12. Nash WL, Gabriel MM. Ex vivo analysis of cholesterol deposition for commercially available silicone hydrogel contact lenses using a fluorometric enzymatic assay. Eye Contact Lens. 2014;40:277-282. doi:10.1097/ICL.0000000000000052

13. Pucker AD, Thangavelu M, Nichols JJ. In vitro lipid deposition on hydrogel and silicone hydrogel contact lenses. Invest Ophthalmol Vis Sci. 2010;51:6334-6340. doi:10.1167/iovs.10-5836

14. Carney FP, Nash WL, Sentell KB. The adsorption of major tear film lipids in vitro to various silicone hydrogels over time. Invest Ophthalmol Vis Sci. 2008;49:120-124. doi:10.1167/iovs.07-0376

15. Luensmann D, Omali NB, Suko A, et al. Kinetic deposition of polar and non-polar lipids on silicone hydrogel contact lenses. Curr Eye Res. 2020:1-7. doi:10.1080/02713683.2020.1755696 
16. Pucker AD, Nichols JJ. A method of imaging lipids on silicone hydrogel contact lenses. Optom Vis Sci. 2012;89:E777-87. doi:10.1097/OPX.0b013e318253dea9

17. Luensmann D, Zhang F, Subbaraman LN, et al. Localization of lysozyme sorption to conventional and silicone hydrogel contact lenses using confocal microscopy. Curr Eye Res. 2009;34:683-697. doi:10.1080/02713680903015900

18. Cheung SW, Cho P, Chan B, et al. A comparative study of biweekly disposable contact lenses: silicone hydrogel versus hydrogel. Clin Exp Optom. 2007;90:124-131.

19. Zhao Z, Naduvilath T, Flanagan JL, et al. Contact lens deposits, adverse responses, and clinical ocular surface parameters. Optom Vis Sci. 2010;87:669-674. doi:10.1097/OPX.0b013e3181ea1848

20. Omali NB, Subbaraman LN, Heynen M, et al. Lipid deposition on contact lenses in symptomatic and asymptomatic contact lens wearers. Cont Lens Anterior Eye. 2020. doi:10.1016/j. clae.2020.05.006

21. McCulley JP, Shine W. A compositional based model for the tear film lipid layer. Trans Am Ophthalmol Soc. 1997;95:79-88; discussion -93.

22. Craig JP, Tomlinson A. Importance of the lipid layer in human tear film stability and evaporation. Optom Vis Sci. 1997;74:8-13. doi:10.1097/00006324-199701000-00014

23. Morgan PB, Woods C, Tranoudis I, et al. International contact lens prescribing in 2019. Contact Lens Spectrum. 2020;35:26-32.

24. Lorentz H, Heynen M, Kay LM, et al. Contact lens physical properties and lipid deposition in a novel characterized artificial tear solution. Mol Vis. 2011;17:3392-3405.

25. Bron AJ, Tiffany JM, Gouveia SM, et al. Functional aspects of the tear film lipid layer. Exp Eye Res. 2004;78:347-360. doi:10.1016/j. exer.2003.09.019

26. Rantamaki AH, Seppanen-Laakso T, Oresic M, et al. Human tear fluid lipidome: from composition to function. PLoS One. 2011;6: e19553. doi:10.1371/journal.pone.0019553

27. McMahon A, Lu H, Butovich IA. The spectrophotometric sulfo-phospho-vanillin assessment of total lipids in human meibomian gland secretions. Lipids. 2013;48:513-525. doi:10.1007/ s11745-013-3755-9

28. Menzies KL, Jones L. In vitro analysis of the physical properties of contact lens blister pack solutions. Optom Vis Sci. 2011;88:493-501.

29. Pinnow DA, Rich TC, Ostermayer FW Jr, et al. Fundamental optical attenuation limits in the liquid and glassy state with application to fiber optical waveguide materials. Appl Phys Lett. 1973;22:527-529. doi:10.1063/1.1654495
30. Keith DJ, Christensen MT, Barry JR, et al. Determination of the lysozyme deposit curve in soft contact lenses. Eye Contact Lens. 2003;29:79-82. doi:10.1097/01.ICL.0000061687.11408.B7

31. Peters K, Millar T. The role of different phospholipids on tear break-up time using a model eye. Curr Eye Res. 2002;25:55-60. doi:10.1076/ceyr.25.1.55.9965

32. Cheng L, Muller SJ, Radke CJ. Wettability of silicone-hydrogel contact lenses in the presence of tear-film components. Curr Eye Res. 2004;28:93-108. doi:10.1076/ceyr.28.2.93.26231

33. Mirejovsky D, Patel AS, Rodriguez DD, et al. Lipid adsorption onto hydrogel contact lens materials. Advantages of Nile red over oil red O in visualization of lipids. Optom Vis Sci. 1991;68:858-864.

34. Peng CC, Fajardo NP, Razunguzwa T, et al. In vitro spoilation of silicone-hydrogel soft contact lenses in a model-blink cell. Optom Vis Sci. 2015;92:768-780. doi:10.1097/OPX.0000000000000625

35. National Center for Biotechnology Information. PubChem Database. 3-Hexanoyl-NBD Cholesterol, $C I D=57370056$. Available from: https://pubchem.ncbi.nlm.nih.gov/compound/3-Hexanoyl-NBDCholestero. Accessed May 11, 2020.

36. National Center for Biotechnology Information. PubChem Database. Cholesteryl oleate, $\mathrm{CID}=5283632$. Available from: https://pubchem. ncbi.nlm.nih.gov/compound/Cholesteryl-oleate. Accessed May 11, 2020 .

37. Sanbar SS, Smet G. Hypolipidemic effect of polyvinylpyrrolidone in man. Circulation. 1968;38:771-776. doi:10.1161/01.CIR.38.4.771

38. Nicolson PC, Vogt J. Soft contact lens polymers: an evolution. Biomaterials. 2001;22:3273-3283. doi:10.1016/S0142-9612(01) 00165-X

39. Luensmann D, Jones L. Impact of fluorescent probes on albumin sorption profiles to ophthalmic biomaterials. J Biomed Mater Res B Appl Biomater. 2010;94:327-336.

40. Walther H, Phan CM, Subbaraman LN, et al. Differential deposition of fluorescently tagged cholesterol on commercial contact lenses using a novel in vitro eye model. Transl Vis Sci Technol. 2018;7:18. doi:10.1167/tvst.7.2.18

41. Tam NK, Pitt WG, Perez KX, et al. Prevention and removal of lipid deposits by lens care solutions and rubbing. Optom Vis Sci. 2014;91:1430-1439. doi:10.1097/OPX.0000000000000419

42. Luensmann D, Heynen M, Liu L, et al. The efficiency of contact lens care regimens on protein removal from hydrogel and silicone hydrogel lenses. Mol Vis. 2010;16:79-92.
Clinical Ophthalmology

\section{Publish your work in this journal}

Clinical Ophthalmology is an international, peer-reviewed journal covering all subspecialties within ophthalmology. Key topics include: Optometry; Visual science; Pharmacology and drug therapy in eye diseases; Basic Sciences; Primary and Secondary eye care; Patient Safety and Quality of Care Improvements. This journal is indexed on PubMed

\section{Dovepress}

Central and CAS, and is the official journal of The Society of Clinical Ophthalmology (SCO). The manuscript management system is completely online and includes a very quick and fair peer-review system, which is all easy to use. Visit http://www.dovepress.com/ testimonials.php to read real quotes from published authors. 